\title{
NUMBER THREE AS A LAW OF SCIENCE. IN PRAISE OF NUMBER THREE
}

\author{
Arkadiusz Maciuk, Antoni Smoluk
}

\begin{abstract}
Comprehension of nature, in the simplest and quickest fashion, boils down to the differentiation of three states. This is probably related to the psychological fact that the human mind grasps only natural numbers from zero to three and the other numbers it calculates. We live in a divalent world created by Aristotle. However not everything can be reduced to two categories: “yes" and "no", because Nature is abundant. The principle of continuity which facilitates understanding is in natural conflict with the binary description of the world. Ever since the times of Aristotle it has been normal to use in science a description of the world that is reduced to two states: "true" and "false". In nature it is more obvious to distinguish three states: low-medium-high or negative-neutral-positive, etc. Man embraces at a single glance sets of three elements at most, and more numerous sets are divided into parts. Binary logic may have a negative impact on the process of teaching and examinations, especially if the tests are used.
\end{abstract}

Keywords: perception of natural numbers, three-valued logic, test exams.

Jel Classification: A20, C60.

DOI: $10.15611 / \mathrm{dm} .2016 .13 .04$.

\section{Introduction}

Three points not located on the same line clearly define the plane. This is one of the axioms of Euclid's Elements and it is empirically verifiable. A tripod - known since time immemorial - does not wobble when set on any surface. Science is based on bivalent logic, separated from philosophy and elevated to the rank of self-discipline by Aristotle. The influence of this philosopher on the world of science is predominant. The binary system is widely used in science and great science operates only based on the concept of truth and falsehood. However, the indiscriminate use of the binary system can have negative consequences, both in the context of didactics (see [Elbow 1993]) or generally understood social sciences [Sztumski 2015], as well as in statistics

Arkadiusz Maciuk, Antoni Smoluk

Department of Mathematics and Cybernetics, Wrocław University of Economics

arkadiusz.maciuk@ue.wroc.pl 
[Hoekstra et al. 2006]. In common parlance and in everyday life in a natural way we distinguish three states: few, medium, many. The tertians set out below are values that are easily grasped by intuition. The aim of the article is to show that the number three is more than a specific measure of the harvest is a rule of nature. Applying this rule, for example during the construction of tests verifying students' knowledge, can have a positive impact on teaching.

\section{In praise of number three}

We know three states of equilibrium: unstable - ball lies on top of the mountain, neutral - ball is on the plane, and stable - the ball is in the hole. The stable equilibrium - like a roly-poly toy - is characterized by its ability to return to its initial state after minor disruptions. The Watt regulator in a steam engine keeps the system running at a constant speed, i.e. homeostasis is a variation of the stable equilibrium. An unstable equilibrium is also stable because the replacement of the ball lying on top of a hill for a balloon lying below the surface of the hill changes an unstable equilibrium into a stable one. Gothic altars are mostly triptychs, triple holes in church choirs are triforia. Number three radiates beauty and harmony. There were three Graces, the Big Three during the Second World War, and so on. In theology the Trinity is probably linked to three attributes of God: Love, Wisdom and Power; the love of the Father, the wisdom of the Spirit and the power of the Son. Philosophers are divided into those of the spirit, the matter and dualists who connect one with another. In common knowledge physics distinguishes three basic states of matter: gas, liquid and solid ${ }^{1}$. A full family has children, parents and grandparents. In most languages, grammar has three quality levels: low, normal and high. Adjectives are graded; a flower may be beautiful, more beautiful, the most beautiful in the whole bouquet. There are also three types of nouns: masculine, neuter, female. Each and every thing and every phenomenon has a beginning, middle and end; every fight starts with skirmish, then there comes a decisive battle and usually ends in the rout of one part and victory of the other. An equilateral triangle is a perfection that is sometimes synonymous with God; that excellence can be seen in the interlacing of the two equilateral triangles forming the Star of David. The popular Russian phrase says "Boh trojcu lubit". St. Paul enumerates faith, hope and love - three states of the human spirit. The threefold repetition of the word represents the highest intensity of the content contained therein. SANCTUS! SANCTUS! SANCTUS!

\footnotetext{
${ }^{1}$ There is the fourth state of the matter, beyond common knowledge: the plasma.
} 
The simplest description of the world is that presenting its three stages: low level of value, medium and high. There is the poor, the middle class and the rich class. Wealth grows exponentially: $y^{\prime}=p y$; this is an equation of the natural growth of capital. Indirectly, it shows that the wealthiest class of people is growing at the same time increasing its assets. In sports there are three possible results of a competition: win, draw and loss. Objectively, so we have the truth, half-truth and falsehood. Professor Father Tischner emphasized the wisdom of the Tatra highlanders who distinguished sacred truth, also truth and untruth. It seems that in science there is only also truth. This condition is well recognized by Łukasiewicz's trivalent logic. There are also modal logics in which one discerns possible states. There is also the body of $Z_{3}=\{0,1,2\}$ of integers modulo 3 residues, whose additive group is isomorphic to a subgroup $T_{3}$ of cube root of one from the wheel group $T$. Number three is the nearest integer number to Euler $e$. This means that the ternary system of writing numbers has a natural advantage that is designed over the commonly used binary system of computing machines. The first computer designed in Wroclaw was meant to work in the ternary system. This system enabled the acceleration of work and shortened the time to perform some arithmetic operations. In the binary system there are only two states: zero - there is no electricity, one - there is electricity. In the ternary account we have three states: zero - no voltage, one - low voltage, two - high voltage. The ternary system fits so naturally in the electrical networks.

Quality is measured by preference - reflexive and transitive relation. The rule of the two-thirds says when the majority relation established for the entire population from individual preferences is again a preference. In order for that to occur every decision should be adopted by the whole population with a probability greater than $\frac{2}{3}$ [Łyko 2000].

\section{Number three - a law of nature}

The largest number which an individual from the Homo sapiens family, as well as some animals, can embrace with one glance is the number three. It is a specific quantitative trait of our brain that sees that there is nothing, i.e. 0 , sees one object, sees a two-piece set, i.e. number 2 , and also sees a threeelement set, i.e. number 3 . We cannot see greater numbers, but we calculate these using partitions. I see, I count, I know. Four is $2+2$, five is $2+3$, six is $3+3$ and so on up to eleven, twelve is a dozen because that means a lot. 
The human brain is such a natural cybernetic system which naturally distinguishes three states. This feature of the mind is a law of nature which is omitted in the teaching of mathematics, as well as in most of the surveys and test exams.

\section{Exams and surveys}

A survey is the preference of the subject, i.e. a reflexive and transitive relation. A friendly survey gives one a choice of up to three possibilities for each question. Also test examination tasks should take into account the perception of this law. Among the responses we distinguish only three states: the correct answer for two points, a possible for one, and a wrong answer for zero points. The probability to pass a test by randomly selecting answers, or without preparation, must be small: in the order of $10 \%, 5 \%$ or less. Probable answers marked with one point give the possibility to extend the advantages of the test with the qualities of an oral exam. This is about including incomplete knowledge into the exam. To determine the border probability of passing an exam by drawing from this, one needs to find the distribution of the results with $n$ tasks. For each task, depending on the response one can get 0,1 , or 2 points. What is the probability distribution of $P_{n}$, when we randomly select $n$ times the variable with values of $0,1,2$ with the same probability, i.e. equal to $\frac{1}{3}$ ? $P_{1}$ is of course a classic distribution, $P_{2}$ is $\left(\frac{1}{9}, \frac{2}{9}, \frac{3}{9}, \frac{2}{9}, \frac{1}{9}\right)$. It is, like all the others, a symmetric distribution which means that the value of 0 in the two draws occurs with a probability of $\frac{1}{9}, 1$ point is obtained with a probability of $\frac{2}{9}$, two points with a probability of $\frac{3}{9}, 3$ with probability $\frac{2}{9}$ and 4 with a probability of $\frac{1}{9}$. Generally we have $P_{n}=\left(p_{n 0}, p_{n 1}, \ldots, p_{n 2 n}\right)$, where $p_{n k}$ is the probability that with the solution of $n$ tasks we get $k$ points. We have $3^{n}$ possibilities, one only needs to calculate the number of possibilities $n_{k}$ giving $k$ points. Then $p_{n k}=\frac{n_{k}}{3^{n}}$, where $k=0,1, \ldots, 2 n$. The values $n_{k}$ are the coefficients of $n$-th power of the function generating $f(x)=1+x+x^{2}$; where $f^{n}(x)=\sum_{k=0}^{\infty} n_{k} x^{k}$ where the coefficients for $k>2 n$ are zero. The generating function is naturally a power series which in this case is reduced to a second degree polynomial. The polynomial $f^{n}$ is of the second degree $2 n$, and its value for $x=1$ is $3^{n}$. Hence, the positive coefficients of the polynomial, which are natural numbers, add up to $3^{n}$. Of course $f^{2}(x)=$ $1+2 x+3 x^{2}+2 x^{3}+x^{4}$, so $p_{23}=\frac{2}{9}$. These values can also be calculated 
without generating function, and using the sequence of vectors $w=\left(w_{1}, w_{2}, w_{3}, \ldots\right)$, which is defined recursively. Let $F(\mathbb{N}, \mathbb{R})$ denote the vector space of all real-valued sequences. The operator $E: F(\mathbb{N}, \mathbb{R}) \rightarrow$ $F(\mathbb{N}, \mathbb{R})$ will be a shift specified by the formula $E(a)=\left(0, a_{0}, a_{1}, \ldots\right)$, where $a=\left(a_{0}, a_{1}, \ldots\right)$. The sequence of vectors $w$ satisfies the recursive equation $w_{1}=(1,1,1,0,0, \ldots), w_{n+1}=w_{n}+E\left(w_{n}\right)+E\left(E\left(w_{n}\right)\right)$; therefore it is $w_{2}=w_{1}+E\left(w_{1}\right)+E^{2}\left(w_{1}\right)$, hence $w_{2}=(1,1,1,0,0, \ldots)+$ $(0,1,1,1,0,0, \ldots)+(0,0,1,1,1,0,0, \ldots)=(1,2,3,2,1,0,0, \ldots)$. The words in sequence $w_{n+1}=\left(w_{n+11}, w_{n+12}, \ldots\right)$ satisfy the equation $w_{n+1 k}=w_{n k}+$ $w_{n k-1}+w_{n k-2}, k=0,1, \ldots$, , where the words with a negative index equal 0 . The above-mentioned symmetry of distributions $P_{n}$ stems from equality $w_{n k}=w_{n 2 n-k}$. Action by operator $E$ is equivalent to the multiplication of the generating function by variable $x$. Operator $I+E+E^{2}$, where $I$ is the the identity operator, corresponds to the generating function $f$.

Table 1. Tabulated distribution $P_{15}$

\begin{tabular}{|c|c|c|c|c|}
\hline$k$ & $2 n-k$ & $w_{15 k}=w_{1530-k}$ & $p_{15 k}=p_{1530-k}$ & Cumulated value \\
\hline 0 & 30 & 1 & 0.00000 & 0.00000 \\
\hline 1 & 29 & 15 & 0.00000 & 0.00000 \\
\hline 2 & 28 & 120 & 0.00001 & 0.00001 \\
\hline 3 & 27 & 665 & 0.00005 & 0.00006 \\
\hline 4 & 26 & 2835 & 0.00020 & 0.00025 \\
\hline 5 & 25 & 9828 & 0.00068 & 0.00094 \\
\hline 6 & 24 & 28665 & 0.00200 & 0.00294 \\
\hline 7 & 23 & 71955 & 0.00501 & 0.00795 \\
\hline 8 & 22 & 157950 & 0.01101 & 0.01896 \\
\hline 9 & 21 & 306735 & 0.02138 & 0.04034 \\
\hline 10 & 20 & 531531 & 0.03704 & 0.07738 \\
\hline 11 & 19 & 827190 & 0.05765 & 0.13503 \\
\hline 12 & 18 & 1161615 & 0.08095 & 0.21598 \\
\hline 13 & 17 & 1477035 & 0.10294 & 0.31892 \\
\hline 14 & 16 & 1704510 & 0.11879 & 0.43771 \\
\hline 15 & 15 & 1787607 & 0.12458 & 0.56229 \\
\hline
\end{tabular}

Source: own elaborations. 
Note. Random variables $h_{n}$ in distribution $P_{n}$ are symmetrical and with a mean $n$ and variance $\frac{2}{3} n$. This is due to the summation of independent random variables. Standardized random variables $h_{n}$ are convergent to the normal distribution with average 0 and variance 1 .

Table 1 shows distribution $P_{15}$; the probability is given with an accuracy of five decimal places. The last column contains the probability of obtaining not less than $k$ points in an exam when the examinee chooses their answers at random. If we want the probability to be small, around $5 \%$ or less, then the threshold number of points should be set at levels greater than 2/3 of the maximum number of points. In our case it will be 21 points; 15 tasks were assumed here, as a semester consists of 15 weeks, which implies that the number is a multiple of 15 lectures [Smoluk 2007, 2007a]. Then one task corresponds to one lecture.

Table 2 lists the number of points $k$ that ensure that with $n$ questions and answers given at random one will not exceed a given level of probability set respectively per mille, percent, five percent, twenty, twenty-five percent and $1 / 3$. For example, with a test consisting of 30 questions determining the pass level at 45 points out of the 60 possible, guarantees that the probability to meet this criterion by randomly selecting the answers is less than one per mille. With twelve questions, in order for the probability to pass the test by selecting the answers at random to exceed the level of 5\%, the pass threshold should be 18 points out of the 24 possible. The second column indicates the number of points possible for $n$ tasks.

Table 2. Border point values

\begin{tabular}{|c|c|c|c|c|c|c|c|}
\hline \multirow{2}{*}{$n$} & \multirow{2}{*}{$\max$} & \multicolumn{7}{|c|}{ Probability less than } \\
\cline { 3 - 8 } & & $1 \%$ o & $1 \%$ & $5 \%$ & $1 / 5$ & $1 / 4$ & $1 / 3$ \\
\hline 5 & 10 & - & 10 & 9 & 8 & 7 & 7 \\
\hline 10 & 20 & 19 & 17 & 15 & 13 & 13 & 12 \\
\hline 12 & 24 & 21 & 20 & 18 & 15 & 15 & 14 \\
\hline 15 & 30 & 25 & 23 & 21 & 19 & 18 & 17 \\
\hline 20 & 40 & 32 & 29 & 27 & 24 & 23 & 23 \\
\hline 30 & 60 & 45 & 41 & 38 & 35 & 34 & 33 \\
\hline 50 & 100 & 69 & 64 & 60 & 56 & 55 & 54 \\
\hline 100 & 200 & 126 & 120 & 114 & 108 & 107 & 105 \\
\hline
\end{tabular}

Source: own elaborations. 
Repetitio est mater studiorum. Distribution $P_{1}$ has a mean that equals 1 and a variance $\frac{2}{3}$, and in general, the distribution $P_{n}$ has a mean that equals $n$ and a variance $\frac{2}{3} n$. The value of variance shows that the majority of the probability concentrates near the mean. Instead of counting recursive relations based on individual values for larger $n$ values, one can use the normal distribution tables. In probabilistics, median made quite a career and quartiles are popular, too; tertians, however, are forgotten - tertians are two points separating the axis of a random variable into three subsets so that the probability of each of them is equal to $1 / 3$. Tertians divide a set of values of a random variable equally-probably into three subsets. In the case of continuous division numbers they are uniquely determined. The probability of obtaining the number of points of the interval $\left[0, T_{n_{1}}\right]$ is the same as the probability of entering into the section $\left[T_{n 1}, T_{n_{2}}\right]$, where $T_{n_{1}}$ and $T_{n_{2}}$ are respectively the first and second thirds of the distribution [Smoluk, Łyko 2015]. From the central limit theorem one can evaluate the value of tertian for distributions $P_{n}$ using tertian $T_{1}$ and $T_{2}$ of a normal distribution with the mean 0 and standard deviation of 1 . Tertian $T_{1}$ of normal distribution $N(0,1)$ is the sequence $\left(\frac{T_{n 1}-n}{\sigma_{n}}\right)$ limit where $\sigma_{n}$ is a standard deviation of the distribution $P_{n}$. Hence the formula:

$$
T_{n 1} \approx \sigma_{n} T_{1}+n \approx n-0.35169 \sqrt{n} .
$$

The accuracy of this formula increases along with the increase of $n$. A similar pattern applies to the second tertian, i.e. $T_{n 2} \approx \sigma_{n} T_{2}+n \approx$ $n+0.35169 \sqrt{n}$.

It is surprising that the level of $2 / 3$ of the total number of examination points - with 15 tasks - is the boundary separating certain knowledge from accidental luck. The likelihood of achieving the level of 2/3 of the maximum number of points decreases with the increase of the number of questions (see Table 2). The limit $g_{n}=1+\left\lfloor\frac{2}{3} 2 n\right\rfloor$, which means that after reaching $k$ number of points the exam is passed when $k \geq g_{n}$, is difficult to cross if the examinee is not prepared and wants to pass the exam using the hit and miss method. The probability of occurrence of $k \geq g_{n}$ decreases to zero with increasing $n$; $2 / 3$ threshold is high. 


\section{Examples of tasks}

In the task below one of the three given answers has to be chosen.

Task 1. In two rows of matrix $A$, the words are different from zero, and the remaining lines are only zero. The row of matrix $A$ is: 0 ; greater than or equal to $1 ; 2$.

Task 2. Given matrix $A$ and matrix $B=A A^{T}$. The row of the matrix is: equal to the rank of the matrix $B$; $\operatorname{det}(B)<0$; $\operatorname{det}(B)>0$.

Task 3. The equality $\operatorname{det}\left(A A^{T}-A^{T} A\right)=0$ is true if and only if matrix $A$ is: square; symmetrical; any.

Task 4. Using Sylvester's claim on the specificity of quadratic form, one can state that the matrix $\left(\begin{array}{ccc}3 & 1 & -1 \\ 0 & 2 & 0 \\ -1 & 0 & 1\end{array}\right)$ is: positively defined; negatively defined; it does not meet the assumptions of the theorem.

Task 5. The cardinal number of difference and finite sets $A$ and $B$ is equal to the cardinal number of set $A$ means that: $B$ is empty; $B$ is a subset of $A$; the sets are disjointed.

Task 6. Function $f: \mathbb{R} \rightarrow \mathbb{R}$ is concave and has a local maximum. This means that $f$ : it has only one extreme point; $f$ has a second derivative; $f$ has a minimum.

Setting up of such a task is not easy because mathematicians are by nature burdened with precision and only distinguish true and false sentences. Mathematical half-truths depend on the object - sometimes it is true, sometimes it is false. Let us consider two similar tasks as an example:

Task 7. The second derivative of the polynomial $a x^{3}+4 x^{2}$ is: the polynomial of the first degree; a polynomial of the second degree; a polynomial of degree no greater than 1 .

Task 8. The third derivative of the function $f: \mathbb{R} \rightarrow \mathbb{R} f(x)=$ $\left(x^{3}-2 x^{2}+x\right)^{2}$ is a polynomial: of the fourth degree; of at most the fourth degree; the third degree, the coefficient of which with the highest power is equal to 5 !

In task 7 the second answer is correct, assuming that parameter $a$ is not equal to zero; the third answer is always correct regardless of the value. In task 8 the second and third answers are correct, the third response is more accurate though. In determining the gradation of tasks to 1 point for the second answer and 2 points for the third answer, one promotes the desired precision in mathematics and accounting capabilities, but this may lead to confusion. The purpose of science is also perfection of the form; the law of science 
itself, as divine perfection, should be dressed in perfect form. A half is either $1 / 2$ or 0.5 or $2^{-1}$ all possible ways of recording are equal, and their use depends on the context. A half is also $17 / 34$, it is $0.4999 \ldots$, and also $\ln \sqrt{e}$, and $\int_{0}^{1} x d x$ etc., etc. These baroque ways of writing numbers have little in common with aesthetic and scientific perfection.

There is no half-truth in mathematics. There are accurate and inaccurate statements. The answer 0.333 is not valid if the right result is $1 / 3$. Answers that are accurate and precise, should be considered as appropriate. In Task 7 a specific polynomial of the third degree is a general answer and a polynomial in the form of $5 ! x^{3}+\cdots$ is the precise answer. Sometimes the divine solution comes in the form of the golden mean, but there are situations when one needs to go down to the precise 'shore' or to the other, intuitive one. Precision in teaching mathematics at lower levels of education may cause harm, as well as the lack of it. If an exercise contains sets $A$ and $B$ and $B$ is a subset of $A$, then for the answer shown in Figure 1 there should be two points awarded, in Figure 2 one point, and for the answer in Figure 3, zero points.

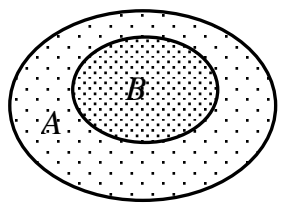

Fig 1. Truth, i.e. 2 points

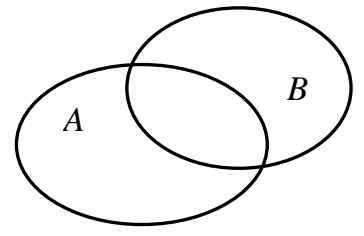

Fig. 2. Half-truth, i.e. 1

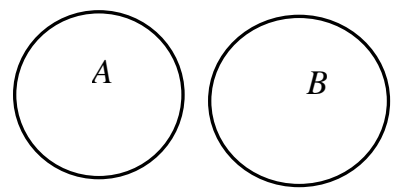

Fig. 3. False, i.e. 0

Source: own elaborations.

\section{Concluding remarks}

Incidentally we would like to suggest a new system of matriculation. Individual items can be considered as tasks; if an examinee takes a test in 6 subjects and receives the results of 0,1 or 2 points, it is enough to pass their final exams by getting 8 points out of the 12 possible. Even if from some of the subjects they get 0 points, they would still graduate provided that from the other subjects they received a sufficient number of points. A secondary school certificate is also intended to prove one's unilateral talents: mathematical, technical, artistic, etc. Forcing students to take exams in all subjects is an educational evil that smothers specific talents. Hugo Steinhaus said that a doctor 
must pass all subjects with at least a satisfactory grade, a lawyer with satisfactory but one of them with flying colors, and a mathematician only needs one subject credited with a very good grade.

\section{Bibliography}

Elbow P. (1993). The uses of binary thinking. Journal of Advanced Composition 12. Pp. 51-78. Hoekstra R., Finch S. Kiers H.A., Johnson A. (2006). Probability Certainty as: Dichotomous thinking and the misuse of p values. Psychonomic Bulletin \& Review 13(6). Pp. 1033-1037.

Łyko J. (2000). Twierdzenia Arrowa a ordynacje wyborcze [Arrow's theorem and electoral law], [in:] A. Smoluk (ed.). Elementy metrologii ekonomicznej [Elements of Economic Metrology], Wydawnictwo Akademii Ekonomicznej we Wrocławiu. Wrocław. Pp. 165-168.

Smoluk A. (2007). Podstawy algebry liniowej. Wydawnictwo Akademii Ekonomicznej we Wrocławiu.

Smoluk A. (2007a). Podstawy analizy matematycznej. Wydawnictwo Akademii Ekonomicznej we Wrocławiu.

Smoluk A., Łyko J. (2015). Próba formalnej definicji dobrobytu [Attempt at the formal definition of prosperity]. Śląski Przegląd Statystyczny 13(19). Pp. 15-27.

Sztumski W. (2015). W niewoli myślenia linearnego $i$ binarnego [In the captivity of linear and binary thinking]. Sprawy Nauki 12(205). 